\title{
Shifting Modal Domains: An Imprecision-Based Account
}

\author{
Peter Klecha \\ University of Chicago \\ klecha@uchicago.edu
}

1 The Problem Discourses like those pointed out by Lewis (1979), which involve modals whose domains appear to shift contextually, have not yet been given a formal treatment in the literature.

a. Yaron: This must be a pen. I'm looking right at it.

b. Itamar: Not so; you could be the victim of a deceiving demon.

A similar example not addressed by Lewis, involving teleological rather than epistemic modality, can be seen here:
a. Alice: I want to go outside, but I don't want to get wet.
b. Bryan: You have to wear a raincoat.
c. Alice: No I don't. I could cover every inch of my skin in duct tape.
d. Bryan: I suppose that's technically true, but you get my drift.

Lewis's basic take on these cases is that they involve a modal being used with a particular domain of admissibility; this domain is then expanded via accommodation to render the original claim false. For descriptive purposes, I will refer to the kind of domain shifting in (2c) as Lewis-shifting; correspondingly, I will refer to the kind of domain restriction that Bryan is employing in (2b) as Lewis-restriction.

L-shifting has some interesting properties which set it apart from other kinds of contextual variability. First is pedantry. In (2c) Alice comes across as pedantic - we can imagine that she could have accepted Bryan's utterance and it would have been perfectly felicitous; compare to (3). However, her conversational move is not uncooperative in the strongest sense, i.e., it does not crash the discourse; compare to (4).

(3) Bryan: You have to wear a raincoat.

Alice: Yeah, that seems right.

(4) Bryan: You have to wear a raincoat.

Alice: \# What? How would a condom help me in this situation?

The second property of L-shifting is a kind of faultless disagreement. Bryan is not just factually mistaken, as indicated by his unapologetic response in (2d). This is important because it means that Bryan's original utterance in $(2 b)$ is in some sense false, but in some sense true. Compare to a genuine case of factual wrongness, in a context where there is an umbrella available, in (5). Bryan cannot use the same unapologetic concession; he must fully concede the wrongness of his previous assertion.

(5) Bryan: You have to wear a raincoat.

Alice: I don't have to. I could just use an umbrella.

Bryan: \#Well, I guess you're technically right. But you see my point.

Bryan': Oh! You're right. I was wrong; wearing a raincoat is not the only option. 
Finally, unidirectionality; Bryan is in some sense forced to go along with Alice's shift, and the shift is also not (easily) reversible; this fact was originally noted by Lewis (1979).

This paper seeks to address L-restriction, rather than providing a formal analysis of the dynamics of L-shifting. However, L-shifting is relevant in as far as what sets L-restriction apart from other kinds of domain restriction is that it can undergo L-shifting. The idea that modal domains are determined contextually is of course not new and is well-accounted for by the theory of anaphoric modal domains (Kratzer 1977, Kratzer 1981, von Fintel 1994, Frank 1997, Klecha 2011). But Lrestriction cannot be captured by an anaphoric theory along those lines, since anaphors never shift in the way seen in L-shifting. (Note that by anaphora I just mean any kind of semantic context dependence, rather than, say, strict familiarity or prior mention.)

I propose that modals' domains are, while crucially anaphorically determined, also subject to (im)precision, relying on the framework established by Lasersohn (1999). On this account, L-restriction is just the effect of imprecision. L-shifting is just precisification, or a raising of the standard of precision in a given discourse. Imprecision, on this account, is a parameter of language use rather than semantic interpretation, thus it is a pragmatic phenomenon. This captures distinctions between L-restriction and the usual kind of context domain restriction, and captures similarities between L-restriction and more canonical cases of imprecision.

2 Imprecision Lewis (1979) raises the issue of "loose talk", though he conflates imprecision with vagueness; I take these phenomena to be distinct, following Lasersohn (1999), Kennedy (2007) and Syrett et al. (2010). In Lasersohn's framework, speakers may felicitously utter strictly false expressions, if they only differ from true expressions in "pragmatically ignorable ways" where what counts as pragmatically ignorable may vary contextually. Consider some examples:

a. Bryan: Everyone's here.

b. Alice: No, not everyone is here. Carissa's on maternity leave.

c. Bryan: Well, sure, you're technically right. But everyone who is expected to come is here.

(7) a. Julia: This table is flat.

b. Martina: It's not flat. Nothing made by humans could truly be flat.

c. Julia: I suppose you're right. But you get my drift.

a. Rebekah: Graham arrived at three.

b. Karlos: No, you're wrong; he arrived at two fifty-nine.

c. Rebekah: Well, right, okay. But you get my drift.

In Morzycki's (2011) analysis this is handled by a parameter called the standard of precision, which is the degree of similarity between what is strictly uttered and potential alternatives. If the standard of precision is set at $d$, then if an alternative is at least $d$-similar to the strict semantic value of the expression under consideration, that alternative is included in the expression's pragmatic halo for $d\left(H_{d}\right)$. If after composition, a sentence's halo includes at least one true proposition, it is then pragmatically "true enough".
a. $\quad H_{d}\left(\llbracket \alpha \rrbracket^{w}\right)=\left\{x \mid x\right.$ is $d$-similar to $\left.\llbracket \alpha \rrbracket^{w}\right\}$
b. $\quad \llbracket \alpha \rrbracket^{w}$ is strictly true iff $w \in \llbracket \alpha \rrbracket^{w} ; \llbracket \alpha \rrbracket^{w}$ is true enough for $d$ iff $\exists p \in H_{d}\left(\llbracket \alpha \rrbracket^{w}\right) . w \in p$ 
This is what is going on in (6a), where Bryan excludes Carissa from the domain of everyone because she doesn't make much of a difference, i.e., the faculty minus Carissa is $d$-similar to the whole faculty. The other cases are analyzed similarly:

$$
\begin{array}{ll}
\text { a. } & H_{d}\left(\llbracket \text { flat } \rrbracket^{w}\right)= \\
& \left\{\ldots, \llbracket \text { nearly flat } \rrbracket^{w}, \ldots, \llbracket \text { very nearly flat } \rrbracket^{w}, \ldots \llbracket \llbracket \text { flat } \rrbracket^{w}\right\} \\
\text { b. } & H_{d}\left(\llbracket \text { three o'clock } \rrbracket^{w}=\right. \\
& \left\{\ldots, \llbracket \text { two fifty nine } \rrbracket^{w}, \ldots, \llbracket \text { three o'clock } \rrbracket^{w}, \ldots, \llbracket \text { three oh one } \rrbracket^{w}, \ldots\right\}
\end{array}
$$

3 L-Restriction is Imprecision The canonical cases of precisification seen above are deeply parallel to L-shifting. This parallel is the primary motivation for pursuing an imprecision-based account of L-restriction. The cases above are just like L-shifting in that they involve pedantry, faultless disagreement, and unidirectionality. Lewis particularly pointed out unidirectionality as a property of both imprecision (his vagueness) and L-shifting, but he did not unify the accounts.

Another point of similarity is that certain modifiers can make truth-conditional what is otherwise pragmatic approximation.
a. He came at 3 .
b. He came at about/approximately/like 3 .

A few of these modifiers can apply to both individual and modal quantifiers, suggesting a strong parallel between the two cases.
a. $\quad\{$ Basically/Practically $\}$ everyone is here.
b. You $\{$ basically/practically $\}$ have to wear a raincoat.

Moreover, certain expressions can serve to raise the standard of precision; these expressions appear with both modal and non-modal expressions.

a. Strictly speaking, not everyone was there.

b. Strictly speaking, you don't have to.

I therefore propose a unified account: LR is just imprecision; LS is just precisification. Thus I argue that in (2) the halo around have to's modal base includes sets which do not contain strictly all the accessible worlds, but rather a subset of them which excludes non-stereotypical/low probability (thus pragmatically ignorable) worlds.

$$
\begin{array}{ll}
\text { a. } & \llbracket \text { alice has to } \text { MB }_{M B} \text { wear a raincoat } \rrbracket^{w}= \\
& \forall v \in M B(w) . \text { wears }(a, r, v) \\
\text { b. } & H_{d}\left(\llbracket \text { alice has to }{ }_{M B} \text { wear a raincoat } \rrbracket^{w}\right)= \\
& \{\forall v \in x(w) . \text { wears }(a, r, v) \mid d \text {-similar }(x, M B)\}
\end{array}
$$

The worlds that can be excluded are those which can be ignored; I argue these are the lowprobability or non-stereotypical worlds. The effect of imprecision on modality should be connected to the notion that humans cannot easily call to mind many of the infinite possibilities that every modal expressions quantifies over. If less likely possibilities come to mind less easily, it stands to reason that imprecision has the effect of excluding less likely possibilities, thus performing a function similar to a stereotypical ordering source. So the effect of imprecision is thus essentially that 
of Kratzer's (1981) stereotypical ordering, but achieved by a general pragmatic principle. This is similar to speaking imprecisely when talking about things like heights or other naturally occurring continuous phenomena that humans cannot under normal circumstances have precise knowledge about. When we say Dave is 6 feet tall it is not just that we know his exact height and choose to be imprecise; rather, speakers almost certainly do not (and cannot reasonably) know the exact height; imprecision allows for an escape from this human limitation.

By understanding L-restriction as imprecision, an analysis can be provided for the recalcitrant case of L-shifting, which has the additional benefit of potentially simplifying the semantics by showing that this is a property of language use rather than conventional meaning.

4 L-Restriction is Not Anaphora In a case where Alice goes along with Bryan's L-restriction, it seems like it could be adequately captured by, say, a stereotypical ordering source a la Kratzer (1981), which is determined anaphorically; but cases where Alice L-shifts show that this Lrestriction cannot be anaphoric. An anaphoric theory of L-restriction would make several incorrect predictions; if the domains of the modals are determined (entirely) anaphorically, their values should be permanently fixed by the context of utterance, not subject to negotiation as in (2).

Determination of the modal base/ordering source is taken to be anaphoric (Frank, 1997, Klecha, 2011), and clearly behaves as such. (15) is clearly an ill-formed discourse because Bryan uses an epistemic modal base for have to; Alice uncooperatively shifts the modal domain to a deontic one.

Bryan: (I just saw him go in there, so) Chuck has to be in the kitchen.

Alice: \# No, he doesn't. He's allowed to be anywhere he wants.

L-shifting cannot just be a case of moving to a new value for an anaphorically determined modal domain, since this is generally not possible.

5 Conclusion Modals, like other expressions, are subject to imprecision. This allows for a straightforward pragmatic analysis of L-restriction and L-shifting, which account for contrasts between L-restriction and anaphoric domain restriction, and for similarities between L-restriction and canonical cases of imprecision.

\section{References}

Frank, A. (1997). Context Dependence in Modal Constructions. Ph. D. thesis, University of Stuttgart.

Gillies, A. S. (2007). Counterfactual scorekeeping. L\&P 30(3), 329-360.

Kennedy, C. (2007). Vagueness and grammar. L\&P 30(1), 1-45.

Klecha, P. (2011). Optional and obligatory modal subordination. Proceedings of Sinn und Bedeutung 15, 365-379.

Kratzer, A. (1977). What 'must' and 'can' must and can mean. L\&P 1(3), 337-355.

Kratzer, A. (1981). The notional category of modality. In Words, Worlds, and Context, pp. 38-74. de Gruyter.

Lasersohn, P. (1999). Pragmatic halos. Language 75(3), 522-551.

Lewis, D. (1979). Scorekeeping in a language game. Journal of Philosophical Logic 8(3), 339-359.

Morzycki, M. (2011). Metalinguistic comparison in an alternative semantics for imprecision. NaLS 19(1), 39-86.

Roberts, C. (1989). Modal subordination and pronominal anaphora in discourse. L\&P 12(6), 683-721.

Syrett, K., C. Kennedy, and J. Lidz (2010). Meaning and context in children's understanding of gradable adjectives. JoS 27(1), 1-35.

von Fintel, K. (1994). Restrictions on Quantifier Domains. Ph. D. thesis, MIT. 